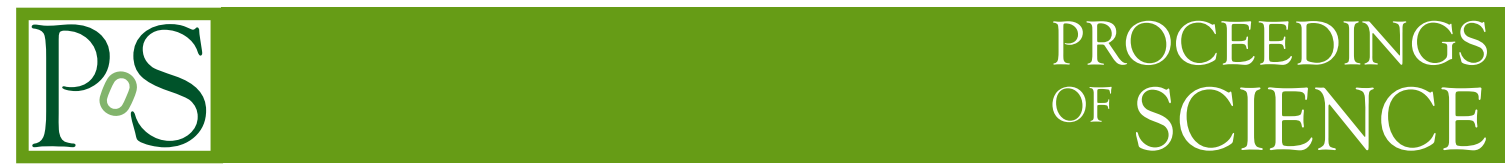

\title{
Standard Model Theory for Collider Processes
}

\section{Giulia Zanderighi ${ }^{* *}$}

University of Oxford and STFC, Theoretical Physics, 1 Keble Road, OXI 3PN Oxford, UK.

E-mail: g.zanderighilephysics.ox.ac.uk

I review the status of today's theoretical description of Standard Model processes relevant for Tevatron and LHC analyses, and of the tools that are used in phenomenological studies. I will also discuss a few recent ideas to further refine our abilities to perform technically challenging calculations.

The 2011 Europhysics Conference on High Energy Physics, EPS-HEP 2011,

July 21-27, 2011

Grenoble, Rhône-Alpes, France

\footnotetext{
*Speaker.

${ }^{\dagger}$ Plenary talk given at EPS.
} 


\section{Introduction}

Today we face many fundamental questions, some of which are driven by experimental data, such as the question about the mechanism of electroweak (EW) symmetry breaking, the nature of dark matter, and the physics associated with the vacuum energy, as well as questions that are driven by theoretical curiosity and ambition, which are in essence propelled by our hope that there is an elegant structure behind what we observe in nature. Questions of the latter type include why there are three generations, what causes the hierarchy of fermion masses and mixing, and how the strong $\mathrm{CP}$ problem is resolved. While questions of the first type do have a definite answer, this is not necessarily the case for the questions of the second type. Therefore those might not even be the right questions to ask. With the start of the LHC we feel that we are at a verge of big changes, the depth of which we can not assess yet. Indeed, the LHC marks the start of a long research program and experiments at the LHC are expected to revolutionize our understanding of the fundamental forces and matter. The LHC will definitely explore the origin of mass and the associated nature of EW symmetry breaking. In the course of this, it might also illuminate the nature of dark matter and the origin of the matter-antimatter asymmetry. It may also explore the physics that underlies the evolution of the early universe. While it is clear that the LHC will not answer all the fundamental questions that we have, the questions we ask now will most likely change after the LHC era. It is therefore really a great and unique time to be a particle physicist, and the "wind of change" was, I believe, particularly strong at this conference.

Since the beginning of its run in 2010, the LHC has been remarkable successful. ATLAS and CMS collected around $45 \mathrm{pb}^{-1}$ in 2010 , more than $1 \mathrm{fb}^{-1}$ by July 2011 , the time of this conference, and more than $5 \mathrm{fb}^{-1}$ by the write-up of these proceedings. Lately, almost every week has marked a new record in instantaneous luminosity. With the 2010 and early 2011 data remarkably, all major Standard Model (SM) processes have already been re-established, including single-top and di-boson production, challenging measurements (because of the small/large cross sections/backgrounds) that have been performed at the Tevatron only in recent years. By now, we have entered a new territory in the search of physics beyond the SM (BSM) with sensitivities already well exceeding those of LEP and the Tevatron.

One important question concerns the role of QCD for LHC measurements and new-physics searches. Understanding how QCD works is essential in order to make accurate predictions for both the signal and background processes. This typically requires complex calculations to higher orders in the perturbative expansion of the coupling constant. Understanding QCD dynamics can however also help reduce backgrounds and sharpen the structure of the signal. This can for instance be achieved by designing better observables, by employing appropriate jet algorithms, by using jetsubstructure, or by exploiting properties of boosted kinematics. Finally, once discovery is made, QCD will be crucial to extract the properties (masses, spins, and couplings) of the new states found. Therefore, at the LHC, no matter what physics you do, QCD will be part of your life.

It is interesting to first recall a recent measurement that was the origin of considerable excitement. In April 2011, CDF reported the observation of a peak in the $m_{j j}$ distribution in $W+$ dijet events [1]. The first measurement had a $3.2 \sigma$ significance, and was based on $4.3 \mathrm{fb}^{-1}$. Subsequently, more data $\left(7.3 \mathrm{fb}^{-1}\right)$ has been analyzed, leading to a significance of more than $4 \sigma$ [2]. Since then, a large number of tentative BSM explanations appeared on the arXiv, along with a few 
SM analysis that address the question of whether this effect can be attributed to a mismodelling of one of the SM backgrounds (in particular single top) $[3,4,5]$. The excitement was curbed shortly before this conference, when D0 announced that it did not confirm the excess seen by CDF [6]. It is yet unclear what the reasons for the discrepancy between CDF and D0 findings are, if any. However, this example demonstrates that even in the case where one identifies a mass peak in the tail of a distribution (a scenario that was considered "an easy discovery") a robust control of SM backgrounds remains mandatory, in particular when the shape of the backgrounds is one of the issues. Currently we have a number of other recent measurements at collider experiments that report a few deviations from the SM predictions. This is for instance the case for the top forward-backward asymmetry measured by both CDF [7] and D0 [8], for the dimuon charge asymmetry measured by D0 [9], for $W+b$ measured by D0 [10], and a few more.

The important question becomes then what the tools at our disposal are to make precise predictions, and whether we have the solid control of backgrounds that is needed in order to claim discoveries. In the following, I will review the current status of our tools, and will discuss a few recent ideas to further improve on the way we perform technically challenging calculations.

\section{Perturbative tools}

The range of physics analyses that one can do at the LHC is very broad. It includes pure instrumental QCD studies, such as measurements of parton densities and inclusive jet cross-section measurements, precision electroweak measurements, Higgs searches, direct and indirect BSM searches, $B$ physics, top physics, diffractive studies and forward physics, and heavy ions physics. Each of these topics includes a vast number of measurements and studies. Yet, there are three things that everybody involved in any of these analyses can not live without: Monte Carlos (MCs), parton distribution functions (PDFs), and jets.

\subsection{MCs and leading order matrix elements}

The first thing "you can not live without" at the LHC are MC generators. Apart from very few exceptions, every analysis at the LHC uses a MC program for the simulation of the signal process, for the backgrounds, for subtracting the underlying event and the non-perturbative contributions, and/or for efficiency studies and modeling of the detector response. The current level of sophistication is such that essentially not a single study relies on Pythia/Herwig alone. It is well understood that in multi-parton processes it is important to describe the multiple hard QCD radiation at least using exact matrix elements, employing for instance Alpgen [11], Madgraph [12], or Sherpa [13].

Since experimental studies rely heavily on all these leading-order (LO) tools, there is continuous progress in their development, and the Herwig/Pythia codes that we have today bear little resemblance to their original version of the '80s. In particular, in Pythia 8.1 [14] (a C++ code) there is a new fully interleaved $p_{\mathrm{t}}$-ordered multi-parton interaction (MPI), initial- and final state evolution (the original mass-ordered evolution is not supported any longer), a richer mix of underlying event processes $(\gamma, J / \Psi$, DY), the possibility to select two hard interactions in the same event, an $x$-dependent proton size in the MPI framework, the full hadron-hadron machinery 


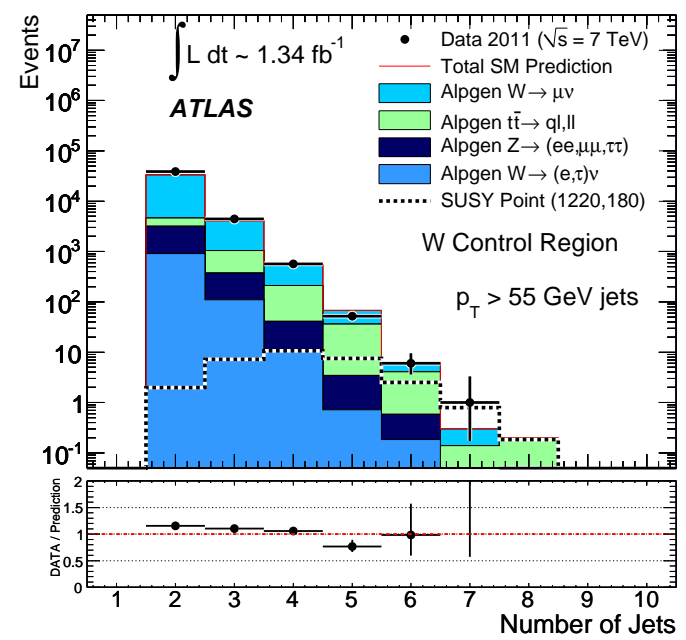

Figure 1: The multiplicity of jets with $p_{T}>55 \mathrm{GeV}$ for events in a $W$ enriched control region at the LHC $(7 \mathrm{TeV})$. Figure taken from [16].

for diffractive systems, several new processes in and beyond the SM, and various other new features. Herwig $++[15]$ (the current version is 2.5.1) has new next-to-leading order (NLO) matrix elements, including weak boson pair production, a colour reconnection model, diffractive processes, additional models of BSM physics, and new LO elements for hadron-hadron, lepton-lepton collisions, and photon-initiated processes. Sherpa [13] (version 1.3) has improved integration routines in Comix, a simplified kinematics reconstruction algorithm of the parton shower (PS), leading to numerically more stable simulations, HepMC output for NLO events and various other improvements/bug-fixes. Madgraph [12] (version 5) has a completely new diagram generation algorithm, which makes optimal use of model-independent information, has an efficient decay-chain package, and a new library for the colour calculations. Altogether, there is continuous, fast progress in various directions. So far, it is amazing how well these tools work, once the normalization is fixed using data. A very recent comparison of data with Alpgen up to six jets (a control region for BSM searches) is shown as an illustration in Fig. 1 [16]. Yet, the devil is in the detail $(\sim 20 \%)$ : for instance in general one expects matrix-element based MCs to work better than pure PSs, but this is not always the case (see e.g. [17]). Altogether, these LO programs will undergo a stress test in the coming years.

\subsection{The NLO revolution}

Theorists like to advertise NLO computations by using the reduction of scale uncertainties in the predictions as an argument, which is meant to reflect the reduction in the theoretical perturbative uncertainty. However, the strongest argument in support of NLO calculations is their past success in accurately describing LEP and Tevatron data. Because of the importance of NLO corrections, an industrial effort has been devoted in the last years to these computations [18]. Recent revolutionary ideas in the way NLO computations are performed include sewing together tree-level amplitudes to compute loop amplitudes (using on-shell intermediate states, cuts, unitarity ideas, ...) [19], the OPP algorithm, an algebraic way to extract coefficients of master integrals by evaluating the amplitudes at specific values of the loop momentum [20], and $D$-dimensional unitarity, a practical 


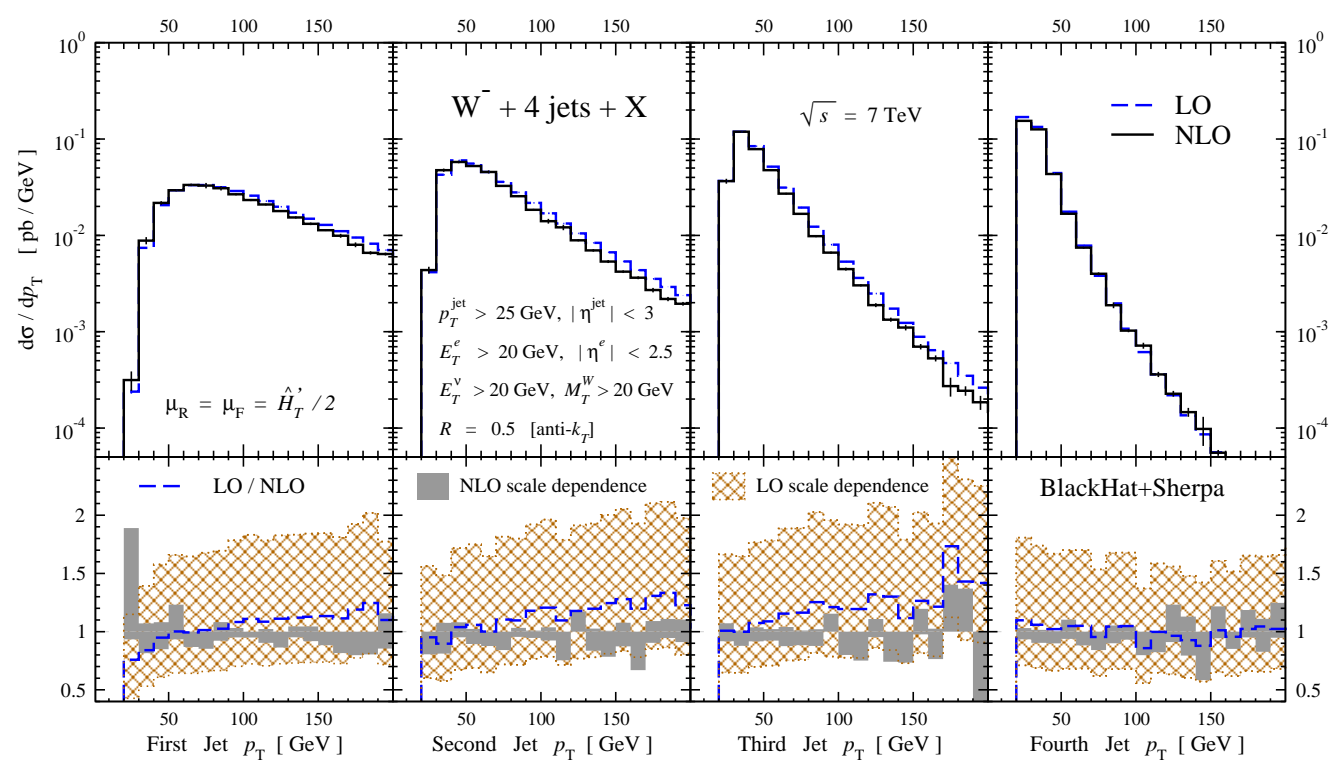

Figure 2: The transverse momentum distribution of the leading four jets in $W^{-}+4$-jet production at the LHC $(7 \mathrm{TeV})$ at LO and NLO. The lower panels show the LO and NLO scale-dependence bands $\left(\hat{H}^{\prime} / 4 \leq\right.$ $\left.\mu_{R}=\mu_{F} \leq \hat{H}_{T}^{\prime}\right)$ normalized to the central NLO prediction $\left(\mu_{R}=\mu_{F}=\hat{H}_{T}^{\prime} / 2\right)$. Figure taken from ref. [36].

numerical tool to evaluate full amplitudes, including the rational part, with unitarity ideas [21]. For a pedagogical review on unitarity methods see [22]. More details on these techniques can be found in the proceedings of Lance Dixon [23].

These methods led in the past 2 to 3 years to a number of $2 \rightarrow 4$ calculations at hadron colliders. These include $W+3$ jets [24, 25], $Z+3$ jets [26], $t \bar{t} b \bar{b}$ [27], $t \bar{t} \rightarrow W^{+} W^{-} b \bar{b}$ [28], $W^{+} W^{+}$ +2 jets [29], $W^{+} W^{-}+2$ jets [30], $t \bar{t}+2$ jets [31], and a few other ones.

Feynman diagram methods have also been applied successfully to $2 \rightarrow 4$ calculations, this is for instance the case for quark-induced $b \bar{b} b \bar{b}$ [32], $t \bar{t} b \bar{b}$ [33], $W^{+} W^{-} b \bar{b}$ [34] production, and a number of VBF processes which are available in the public code VBFNLO [35]. Note that only a few years ago, performing this type of calculation with Feynman diagrams was considered an impossible task.

Given that both Feynman diagram and unitarity based methods allowed us to compute $2 \rightarrow 4$ processes at NLO in QCD, it might be unclear where the revolution advocated in the heading of the subsection lies in. The revolution, I believe, is not yet in the applications that we see today, rather in the prospect for low-cost fully computer-automated NLO calculations even beyond $2 \rightarrow 4$ in the near future. Indeed, two $2 \rightarrow 5$ processes have already been computed at NLO, namely $W$ +4 jets [36] and $Z+4$ jets [37]. ${ }^{1}$ Fig. 2 illustrates in the case of $W^{-}+4$ jets the typical effect of including NLO corrections: one obtains a considerable reduction of the scale uncertainty, and, for some distributions, a change in shape. As far as the full automation is concerned, let me highlight only two interesting approaches. The first one [38] is a method based on Feynman diagrams, it uses the OPP procedure for the virtual calculation, and the FKS subtraction of divergences, together with

\footnotetext{
${ }^{1}$ In both cases the leading colour approximation has been used, and six-quark processes have been neglected. Both approximations are expected to give rise to very small (percent) corrections only.
} 

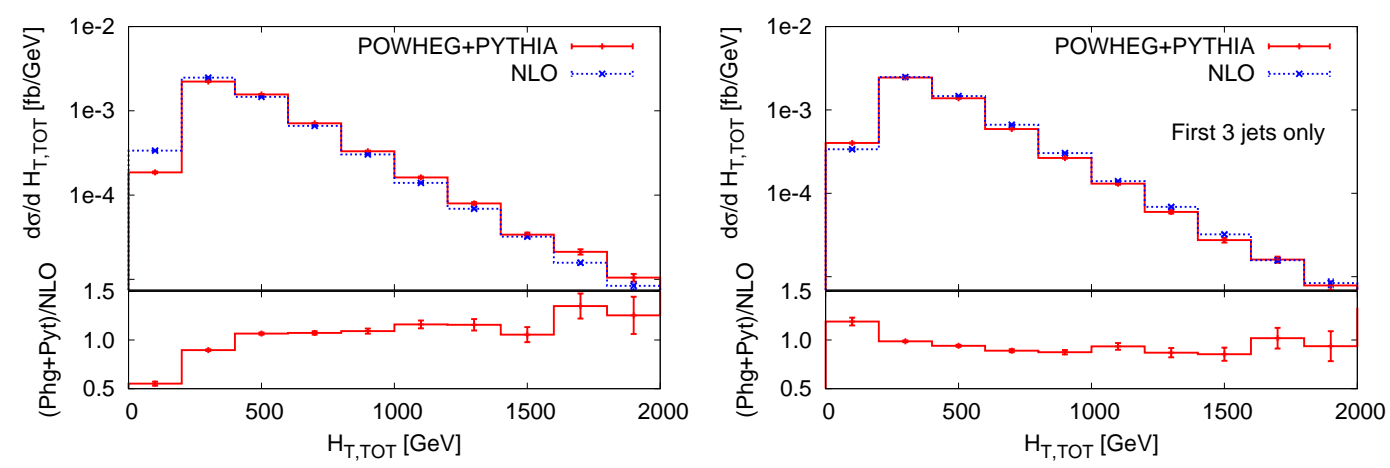

Figure 3: Comparison of NLO and POWHEG+PYTHIA results for the $H_{\mathrm{T}, \mathrm{TOT}}$ distribution in the process $W^{+} W^{+}+2$ jets at the LHC $(7 \mathrm{TeV})$, when all jets are included in the definition of $H_{\mathrm{T} \text {,TOT }}$ (left pane), and when only the three hardest jets are included (right pane). Figure taken from ref. [43].

clever and efficient procedures to deal with instabilities. More improvements and refinements are to be expected soon. At present there is no public code, instead the idea is to provide $N$-tuples. The second approach, HELAC-1LOOP [39], is a program that evaluates numerically QCD virtual corrections to scattering amplitudes. It is based on the OPP technique and the HELAC framework. The public program is part of the HELAC-NLO framework that allows for a complete evaluation of QCD NLO corrections.

\subsection{Merging NLO and Parton Showers}

While NLO predictions provide relatively accurate results for inclusive cross sections, they do not furnish an exclusive description of the final state that can be compared with actual particles in the detectors, as MC programs do. It is therefore useful to combine the best features of both approaches. Two public frameworks exist for this purpose, namely MC@NLO [40] and POWHEG [41]. These tools are almost 10 years old now, and since their conception a long list of processes has been implemented in both frameworks.

In particular, recently the POWHEG BOX was released [42], which is a general framework for implementing NLO calculations in shower MC programs according to the POWHEG method. The user only needs to provide a simple set of routines (Born, colour-correlated Born, virtual, real, and phase space) that are part of any NLO calculation.

The first $2 \rightarrow 4$ process that has been implemented in the POWHEG BOX is $p p \rightarrow W^{+} W^{+}+2$ jets [43]. This is a relatively simple $2 \rightarrow 4$ process since the cross section is finite without any cut on the jets. As expected, for inclusive observables there are only minor differences between pure NLO and POWHEG+PS, but for exclusive observables, depending on the details of the observable definition, there can be important differences. This is shown in Fig. 3 for two different definitions of $H_{\mathrm{T}, \mathrm{TOT}}=\sum_{j} p_{\mathrm{t}, \mathrm{j}}$, the transverse energy of the event. From the figure it is clear that if only the three hardest jets are included in the definition of $H_{T}$, the corrections from the PS are very moderate (right pane), but if all soft jets present in the event are included, then additional radiation from the PS can alter the distribution substantially (left pane).

aMCNLO is a novel approach to a complete event generation at NLO. It has been used for the calculation of scalar and pseudo-scalar Higgs production in association with a $t \bar{t}$ pair [45], 


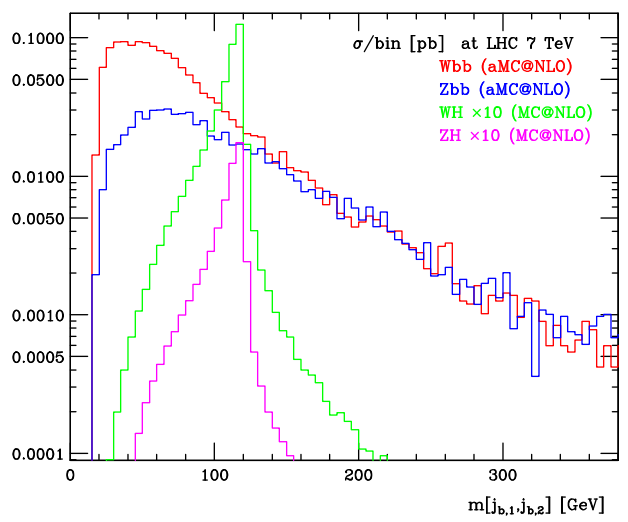

Figure 4: Invariant mass of the pair of the two leading $b$-jets for $W b \bar{b}, Z b \bar{b}, W H(\rightarrow \ell v b \bar{b}), Z H\left(\rightarrow \ell^{+} \ell^{-} b \bar{b}\right)$ at the LHC ( $7 \mathrm{TeV})$, the latter two are rescaled by a factor of ten. Figure taken from ref. [44].

$W / Z b \bar{b}$ [44] and $W+$ dijet production [46]. Fig. 4 shows an application to Higgs searches of the $W / Z b \bar{b}$ calculation: the invariant mass of the pair of the two leading $b$-jets, for the processes $W b b$, $Z b b, W H$, and $Z H$. The figure illustrates a case where signals and irreducible backgrounds are computed with the same accuracy. As yet, no public code is available, instead the idea is to provide ready-to-shower events.

\subsection{MENLOPS and LOOPS im}

MENLOPS $[47,48]$ is a method to further improve on NLO+PS predictions with matrix elements involving more partons in the final state. For example, for $W$ production it includes, as in MC@NLO or POWHEG, $W$ production at NLO, the PS, but also $W+1,2,3, \ldots$ jets using exact matrix elements. Roughly speaking, it uses a jet-algorithm to define two different regimes, and then corrects the 1-jet fraction using exact matrix elements and the 2-jet fraction using the NLO $K$-factor. This achieves NLO quality accuracy for inclusive quantities but an improved sensitivity to hard radiation and multi-parton kinematic features.

A further recent theoretical development is LoopSim. If one considers the process $W+1$ jet, the three observables $p_{\mathrm{t}, \mathrm{Z}}, p_{\mathrm{t}, \mathrm{j}}$, and $H_{\mathrm{T}, \mathrm{jets}}=\sum_{j} p_{\mathrm{t}, \mathrm{j}}$ are identical at LO. However, as illustrated in Fig. 5, at NLO $p_{\mathrm{t}, \mathrm{Z}}$ has a moderate $K$-factor $(\lesssim 2), p_{\mathrm{t}, \mathrm{j}}$ has a large $K$-factor $(\sim 5)$ and $H_{\mathrm{T}, \text { jets }}$ has a giant $K$-factor $(\sim 50)$. The very large $K$-factors in the last two observables is due to the fact that the NLO result is dominated by configurations where there are two hard jets and a soft $W$ (these are enhanced by electroweak logarithms), additionally there is an important enhancement coming from incoming $q q$ channels. LoopS im [49] is a procedure that uses a sequential algorithm, close to the Cambridge/Aachen one, to determine the branching history, "loops" over soft particles (i.e. they are removed from the event and the residual event is adjusted), and it uses a unitary operator to cancel divergences. In essence, this is a way to extend a calculation that is exact at a given order in perturbation theory, in an approximate way to higher orders. The procedure is expected to be more accurate the larger the corresponding $K$-factor is. One might expect other extensions of the MLM/CKKW matching procedure along the same lines as MENLOPS and LoopSimin the near future. 

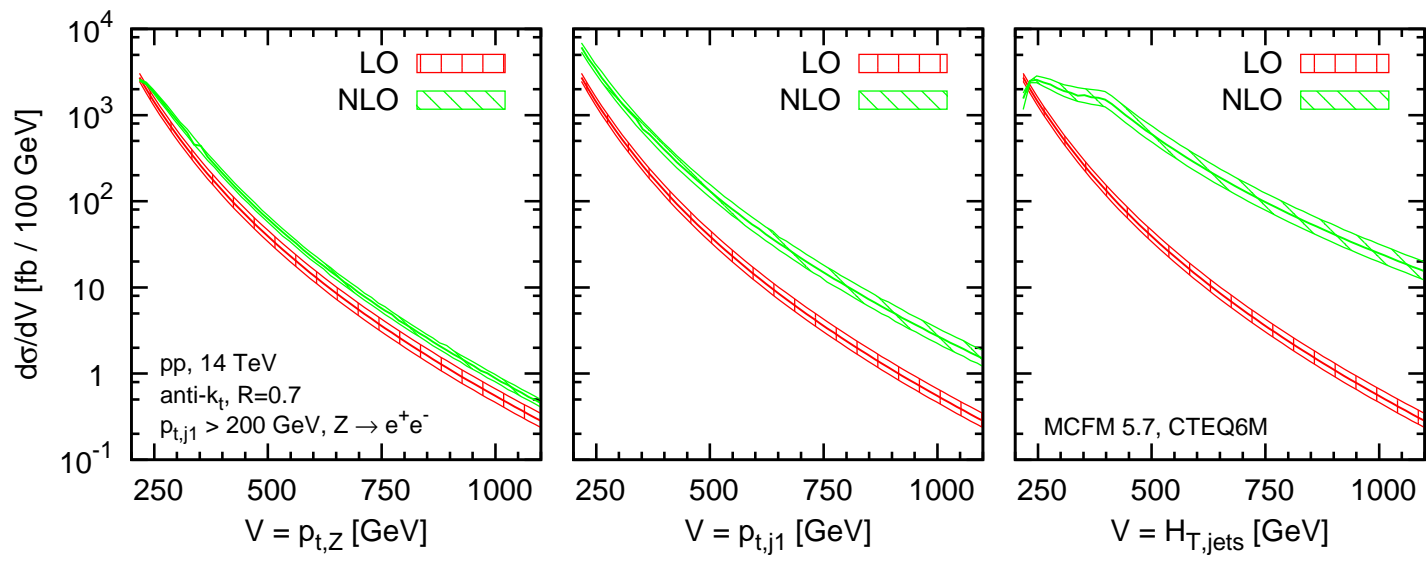

Figure 5: The LO and NLO distributions for three observables in Z+jet production that are identical at LO: the $\mathrm{Z}$ transverse momentum (left), the $p_{t}$ of the hardest jet (middle), and the scalar sum of the transverse momenta of all the jets, $H_{\mathrm{T}, \mathrm{jets}}$ (right). The bands correspond to the uncertainty from a simultaneous variation of $\mu_{R}=\mu_{F}$ by a factor of two either side of a default $\mu=\sqrt{p_{t, j 1}^{2}+m_{Z}^{2}}$. Figure taken from ref. [49].

\subsection{Drell Yan}

Drell Yan is the best known process at the LHC: it has been computed through NNLO in QCD, fully differential in lepton momenta including spin-correlations, EW corrections, finite-width effects, and $\gamma^{*} / Z$ interference. State-of the art codes are described in [50, 51]. Calculations to allorders also exist, for instance the NNLL transverse momentum resummation [52] and soft gluon resummation are also available [53]. These precise perturbative calculations have been available for some time, and now that precise LHC data has been compared to those predictions, one can not but praise the impressive agreement between NNLO theory and experiment (see e.g. Fig. 6 and [54]). In particular, not only cross sections have been measured, but also $W / Z$ properties have been probed, as well as anomalous couplings in di-boson production (see e.g. [55]). More details can be found in the proceedings of Juan Alcaraz [56].

\subsection{Top Quark}

The top is the most interesting SM quark. Its large mass implies a large Yukawa coupling, which causes the top to be a prominent decay product in many BSM models. LHC data have already been successfully compared to approximate NNLO predictions [58, 59], however various approximate NNLO predictions, based on a threshold resummation, do not fully agree within quoted uncertainties $[60,61,62,63]$. Therefore a full NNLO calculation is highly desirable. A better perturbative control of the top-quark pair production cross section is also important to further constrain gluon PDFs, to have an accurate extraction of the top mass from the cross section, and to improve our perturbative control over the $t \bar{t}$ forward-backward asymmetry. In fact, an almost $3 \sigma$ deviation from the SM is observed by $\mathrm{CDF}$, which becomes a $4.2 \sigma$ effect in the high-mass region, $M_{t \bar{t}}>450 \mathrm{GeV}$ [7]. The large inclusive asymmetry has been seen both by CDF and D0 [8], while the rise in the spectrum of the asymmetry is not confirmed by D0. One also has to bear in mind that $t \bar{t}$ production is a difficult measurement given the presence of neutrinos in the final state, the combinatorics in the reconstruction of the tops, and the limited statistics at the Tevatron. 


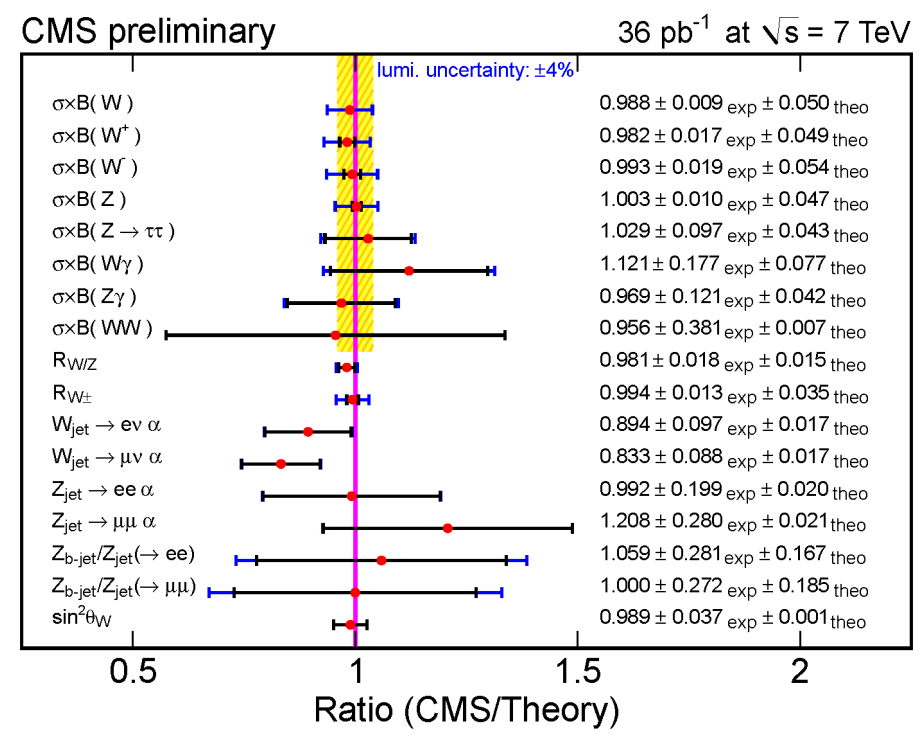

Figure 6: Comparison of NNLO theory and CMS data for Drell Yan observables. Figure taken from ref. [57].

Nevertheless, various suggestions have been made recently to explain the asymmetry in terms of BSM physics, but all proposals face the problem that they have to preserve the good agreement with the symmetric $t \bar{t}$ observables, respect dijet bounds and/or must evade the stringent limits on like-sign top production. Fervid activity is therefore currently devoted towards a complete NNLO calculation of $t \bar{t}$ production (see [64] and references therein).

\subsection{A few theoretical issues in Higgs production}

Possibly the most awaited result at this conference concerned the status of Higgs searches at the LHC. Results from the first $1 \mathrm{fb}^{-1}$ of data, presented at EPS 2011, were rich of hints of excesses. Unfortunately, the second $1 \mathrm{fb}^{-1}$ analyzed since then seems to be lacking any excess. Therefore, the analyses of the next $3 \mathrm{fb}^{-1}$ already collected this year are awaited very eagerly. Currently, ATLAS and CMS are able to individually exclude the region $(145-460) \mathrm{GeV}$ (with an island around 300 $\mathrm{GeV}$ that is not formally excluded yet, but close to being disfavoured), and the next focus will be on the $(114-145) \mathrm{GeV}$ region.

At the LHC, the Higgs is mainly produced via an intermediate top loop in gluon-gluon fusion. The urge to understand the EW symmetry breaking led in the past years to the computation of the most advanced theoretical predictions for this process. For instance we now know the main $g g \rightarrow H$ production mechanism including NLO corrections with exact top and bottom quarks in the loop [65], NNLO corrections in the large $m_{t}$ limit [66, 67, 68], electroweak corrections [69], mixed QCD-EW corrections [70], and resummation of large logarithms possibly with $\mathrm{N}^{3} \mathrm{LO}$ soft effects $[71,72,73,74]$. Furthermore, the most advanced codes $[75,76]$ allow for fully exclusive decays of the Higgs to $\gamma \gamma, W^{+} W^{-} \rightarrow e^{+} v e^{-} \bar{v}$, and $Z Z \rightarrow 4 l$. A similar accuracy has been reached recently also in associated $V H$ production where, because this process is an important one if the Higgs is light, the decay of the Higgs into $b \bar{b}$ has been considered [77]. 


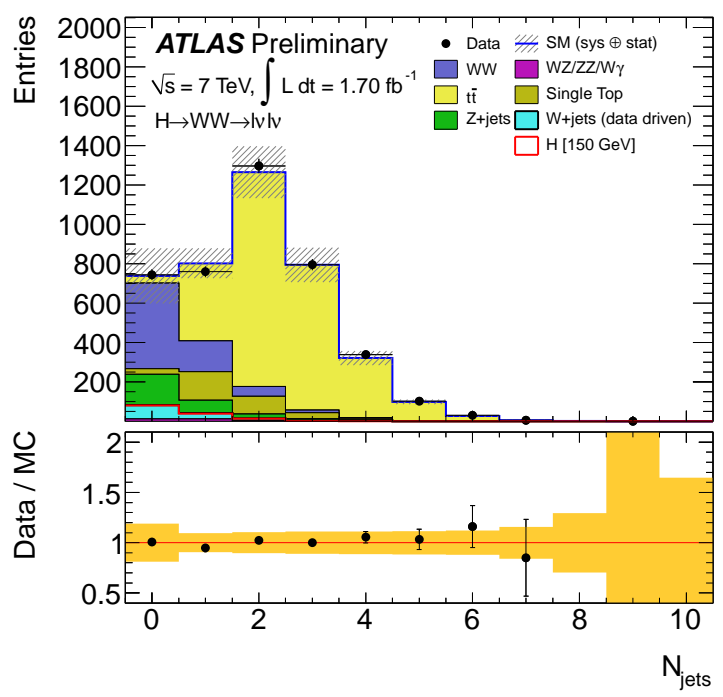

Figure 7: Multiplicity of jets with $p_{T}>25 \mathrm{GeV}$ after basic Higgs search cuts. The lower part shows the ratio between the data and the background expectation from $\mathrm{MC}$, with the yellow band indicating the total systematic uncertainty in the normalization (but not the shape) of the various components. The signal is shown for $m_{H}=150 \mathrm{GeV}$. Figure taken from ref. [81].

Given the high accuracy with which gluon-gluon-fusion has been computed, it is interesting to ask what is the actual theoretical uncertainty on this process. Unfortunately, there is today no consensus on this question. Some more conservative estimates quote errors of the order of 40\% [78] (at the Tevatron) and similar uncertainties at the LHC, while other studies suggest that the perturbative uncertainty is considerably smaller. Assigning a correct theoretical error is very important when claiming an exclusion or an excess, and, at a later stage, when making measurements of the Higgs-boson couplings, which is the only way to identify the precise nature of the Higgs boson and EW symmetry breaking. Yet, even for the main Higgs-production channel there are still some controversies and subtleties. Most controversies have to do with how different sources of errors should be combined, others concern the question of how to assign/interpret the perturbative uncertainties. I will illustrate here just two of these issues.

The soft logarithms appearing in cross sections can be resummed using an effective theory approach. Performing such a calculation requires an introduction of a matching scale, where the full and effective theory amplitudes must agree. It is well-known that choosing a time-like (i.e. complex) matching scale effectively resums $\pi^{2}$ enhanced terms. In [79] it is suggested that this procedure improves the convergence of the perturbative expansion significantly, and reduces the uncertainty of the perturbative (NNLO) prediction. This approach is criticized in [80] with the arguments that $\pi^{2}$ are just numbers, so that there is no formal limit in which they dominate, and that only one class of $\pi^{2}$ terms is resummed (those that arise from the gluon form factor), but not all of them. In this context, one has to mention that perturbative QCD is often about pushing approximations beyond their formal limit of validity, and that a given approach should be judged by seeing how well it fares in practice.

The second issue I would like to mention here has to do with a jet-veto in Higgs searches. As 
can be seen from Fig. 7, in Higgs searches one needs to impose a jet-veto to get rid of the large top background. Higgs production is then studied in 0-, 1-, 2-jet bins separately in order to maximize the sensitivity. Currently, ATLAS uses $p_{\mathrm{t} \text {,veto }}=25 \mathrm{GeV}$, while CMS employs $p_{\mathrm{t} \text {,veto }}=30 \mathrm{GeV}$ in their Higgs searches.

In [82] the inclusive, NNLO Higgs production cross section at the Tevatron is split into 0-,1-jet exclusive, and 2-jet inclusive components

$$
\frac{d \sigma_{\mathrm{tot}}}{\sigma_{\mathrm{tot}}}=66.5 \%{ }_{-9 \%}^{+5 \%}(0-\text { jet })+28.6 \%_{-22 \%}^{+24 \%}(1-\mathrm{jet})+4.9 \%_{-41 \%}^{+78 \%}(\geq 2-\text { jets })=[-14.3 \%+14.0 \%] \text {. }
$$

The errors denote the scale uncertainty that is obtained by varying the renormalization and the factorization scale together around a central value $m_{\mathrm{H}}=160 \mathrm{GeV}$ by a factor of two. In an NNLO calculation of inclusive Higgs production, only the 0 -jet bin is known at NNLO, while the 1-jet bin is know at NLO and the 2-jet bin is computed at LO only. Therefore is it not surprising that the relative errors increase with the number of jets. In [82] one can also find a detailed discussion of why it is not appropriate to use the standard scale variation as an estimate of the perturbative uncertainty for the 0-jet bin cross section (Fig.1 of [83] also shows that for a particular choice of $p_{\text {T,veto }}$ one obtains a vanishing scale uncertainty band in the 0 -jet bin). The numbers in eq. (2.1) were updated by Campbell et al. in [84] who evaluated the 2-jet bin contribution at NLO. The effect of this addition was a slight change in all relative numbers, and, mainly, a decrease in the perturbative uncertainty of the 2 -jet bin,

$$
\frac{d \sigma_{\mathrm{tot}}}{\sigma_{\mathrm{tot}}}=60 \%_{-9 \%}^{+5 \%}(0-\mathrm{jet})+29 \%_{-23 \%}^{+24 \%}(1-\mathrm{jet})+11 \%_{-31 \%}^{+35 \%}(\geq 2 \mathrm{jets})=[-15.5 \%+13.8 \%] .
$$

From eq. (2.2), it is evident that the scale uncertainty is smaller for the exclusive measurement with 0 -jets, than the one of the fully inclusive measurement. To explain this feature, Stewart and Tackmann recall that there are two mechanisms at work in the 0-jet cross section [85]: there is a large $K$ factor from perturbative higher orders, as well as large negative logarithms $-\alpha_{s} C_{A} / \pi \ln ^{2} M_{H} / p_{\mathrm{t} \text {,veto }}$ that become more important the smaller $p_{\mathrm{t} \text {,veto }}$ is. They therefore suggest that the error on the 0 -jet bin should be computed taking into account the correlation between jet-bins, i.e. the error from the 0 -jet cross section is computed from the relation $\sigma_{0}=\sigma_{\text {incl }}-\sigma_{\geq 1-\text { jet }}$. One obtains then simply $\Delta^{2} \sigma_{0}=\Delta^{2} \sigma_{\text {incl }}+\Delta^{2} \sigma_{\geq 1-\text { jet }}$. The effect of this is illustrated in Fig. 8. While this procedure is certainly more conservative than a conventional scale variation, it is clear that to reduce the uncertainty on the jet-veto cross section, a resummation of large logarithms involving the ratio $p_{\mathrm{t}, \text { veto }} / M_{H}$ is required. Currently, only resummation for quantities related to the jet-veto exist, e.g. for $p_{\mathrm{T}, \mathrm{Higgs}}$ [86] or for the beam-thrust [87]. Both observables are however not the ones used in current Higgs searches. Furthermore the beam thrust has the drawback that it receives very large non-perturbative corrections, as can be easily seen by running a PS program at parton or hadron level.

\section{Parton distribution functions and $\alpha_{S}$}

PDFs are the second thing you can not live without, if you work on LHC physics. Huge effort is devoted today in understanding differences and improving the theoretical and statistical 

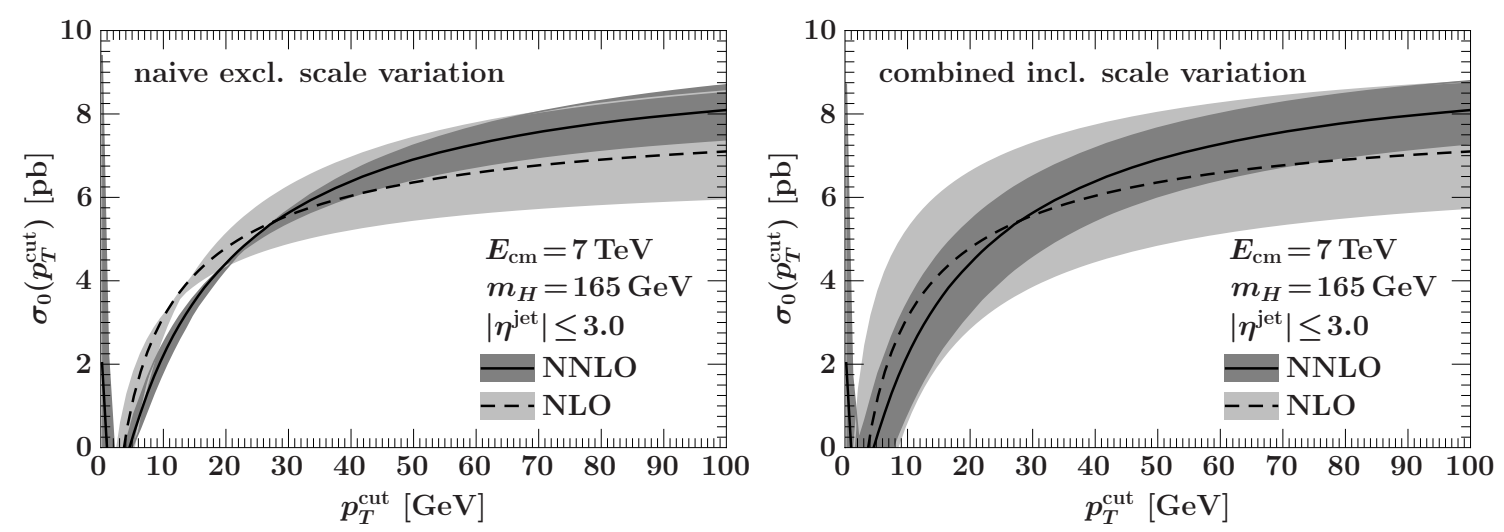

Figure 8: Fixed-order perturbative uncertainties for $g g \rightarrow H+0$ jets at NLO and NNLO for the LHC ( $7 \mathrm{TeV}$ ). On the left, the uncertainties are obtained from the naive scale variation in $\sigma_{0}\left(p_{\mathrm{T}}^{\text {cut }}\right)$ between $\mu=m_{H} / 4$ and $\mu=m_{H}$. On the right, the uncertainties are obtained by independently evaluating the scale uncertainties in $\sigma_{\text {tot }}$ and $\sigma_{\geq 1}\left(p_{\mathrm{T}}^{\text {cut }}\right)$ and combining them in quadrature. Figure taken from ref. [85].

treatment of PDFs. This activity is reflected in new PDFs sets being released by various groups [88]. The main focus of all groups is now directed towards NNLO PDFs sets, an improvement in the treatment of heavy quarks, an introduction of flexible parametrizations, a more dynamic tolerance, and, of course, towards the inclusion of more data in the fits. Discussions are ongoing that try to clarify whether discrepancies between different PDFs are due to the inclusion of different data sets. For instance, there is no full consensus on what impact of the Tevatron jet data has on gluon distributions at the LHC.

Fig. 9 shows the uncertainty on three LHC benchmark processes $\left(Z, W^{+}\right.$, and $t \bar{t}$ from the left to the right) coming from using different PDFs or a different value of $\alpha_{s}$, at NLO and at NNLO. Differences are due to the inclusion of different data in the fits, due to a different methodology (e.g. the parametrization), due to a different treatment of heavy quarks, and due to a different default value of the coupling constant. In particular, it is remarkable how much benchmark processes depend on the value of $\alpha_{s}$. The preliminary 2011 average value of $\alpha_{s}$ is $\alpha_{s}=0.1183 \pm 0.0010$ [89]. It is interesting to note that the value barely changed compared to the 2009 number $\left(\alpha_{s}=0.184 \pm 0.0007\right)$ [90], but that the uncertainty on it increased. This is due to the inclusion of new data in the fits which tend to move the average value in opposite directions. An open issue today, in the combination of the various measurements to produce a world average for $\alpha_{s}$, is the treatment of outliers that have very small errors. This is the case for the extraction of $\alpha_{s}$ from thrust computed at $\mathrm{N}^{3} \mathrm{LL}$ including power corrections using SCET [91], for the number obtained from $\tau$-decays in [92], and for the hadronic event shapes in $e^{+} e^{-}$collisions at OPAL using NNLO+NLLA theoretical predictions [93], just to quote the most important cases.

New processes added to the world average since 2009 include inclusive jets at the Tevatron [95], the $e^{+} e^{-} 3$-jet rate which is know to NNLO [96], and $e^{+} e^{-} \rightarrow 5$ jets, which is now known at NLO [97]. The use of the Tevatron jet data in this context is particularly interesting: while the error on $\alpha_{s}$ from this extraction is not particularly small, this measurement and the sensitivity of benchmark processes on the value of $\alpha_{s}$ shown in Fig. 9 raises the question of whether it is possible to make competitive measurements of $\alpha_{s}$ at the LHC. The extraction of the value of the coupling 

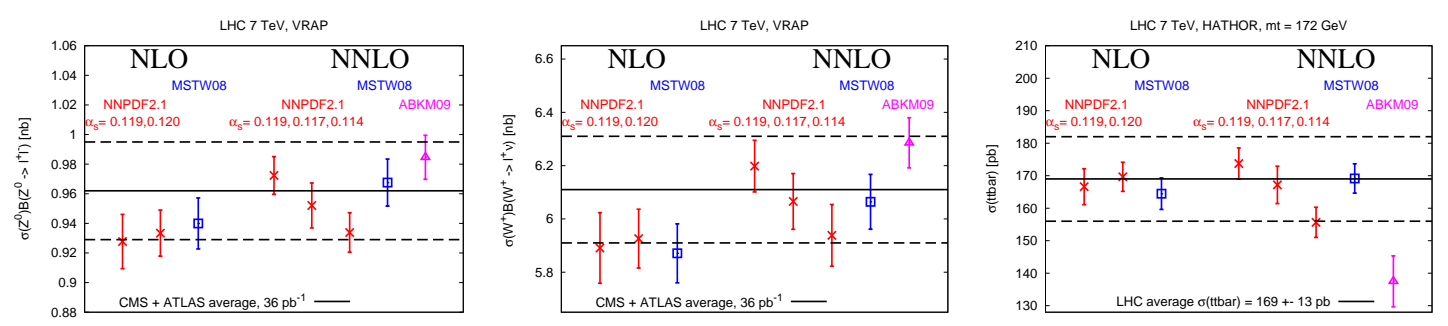

Figure 9: The total cross-section for $Z, W^{+}$, and $t \bar{t}$, at the LHC $(7 \mathrm{TeV})$ for NNPDF2.1 with $\alpha_{s}\left(M_{Z}\right)=$ $0.119\left(\mathrm{NLO}\right.$ and NNLO), $\alpha_{s}\left(M_{Z}\right)=0.120(\mathrm{NLO})$ and $\alpha_{s}\left(M_{Z}\right)=0.114,0.117$ (NNLO), MSTW08 with $\alpha_{s}\left(M_{Z}\right)=0.1202(\mathrm{NLO})$ and $\alpha_{s}\left(M_{Z}\right)=0.1171(\mathrm{NNLO})$, and ABKM09 with $\alpha_{s}\left(M_{Z}\right)=0.1135 \pm 0.0014$ (NNLO). Uncertainties shown correspond to one sigma. The band corresponds to the combination of CMS and ATLAS measurements. Figure taken from ref. [94].

constant at hadron colliders must take into account that PDFs themselves do depend on $\alpha_{s}$. A viable possibility then is to consider appropriate ratios (e.g. $W /(Z+(n+1)$ jets $) /(Z /(W+n$ jets $)))$.

\section{Jet algorithms}

Jet algorithms are the third thing you can not live without, if you do LHC physics. For a long time, infrared (IR) unsafe algorithms were used at the Tevatron, with several "patches" to minimize the effect of the IR-unsafety. At the LHC, both ATLAS and CMS have adopted as default the anti$k_{t}$ algorithm [98]. Given that this algorithm was proposed only three years ago, it shows how flexible experimentalists are today in adopting new, successful ideas. ${ }^{2}$ Using this algorithm both collaborations have already explored scales up to $4 \mathrm{TeV}$ and could place constraints on various BSM models, in particular those models that would give rise to a resonance in the $M_{j j}$ distribution (such as massive coloured bosons, black-holes, ...).

Other IR-safe algorithms like the Cambridge-Aachen or SISCone are in use as well. These are particularly useful for studies which exploit the fact that when a massive boosted object decays, it gives rise to a "fat jet" with a non-trivial jet-substructure. Looking at the internal structure of these jets using jet-grooming techniques like filtering, pruning or trimming has a huge potential for making discoveries "easier" [99]. These techniques have a big gain in sensitivity over traditional methods, but one might lose many events when imposing strict kinematical cuts and requiring a boosted regime. The potential of these studies has been demonstrated in several examples [99]. However sophisticated jet studies are still a young field, and as of now there are no precise rules on how to make discoveries easier. What is impressive, is that even these very new techniques are already being used at the LHC. At this conference we saw for instance a study of the single hadronic jet mass in a boosted regime, an observable relevant for $W H(\rightarrow b b)$. In Fig. 10 the $Z$ peak coming from $W Z(\rightarrow b b)$ is evident and so these very first results seem very promising.

\footnotetext{
${ }^{2}$ A minor downside to this is that ATLAS and CMS use a different radius - the choices for ATLAS are 0.4 and 0.6, while for CMS they are 0.5 and 0.7 .
} 


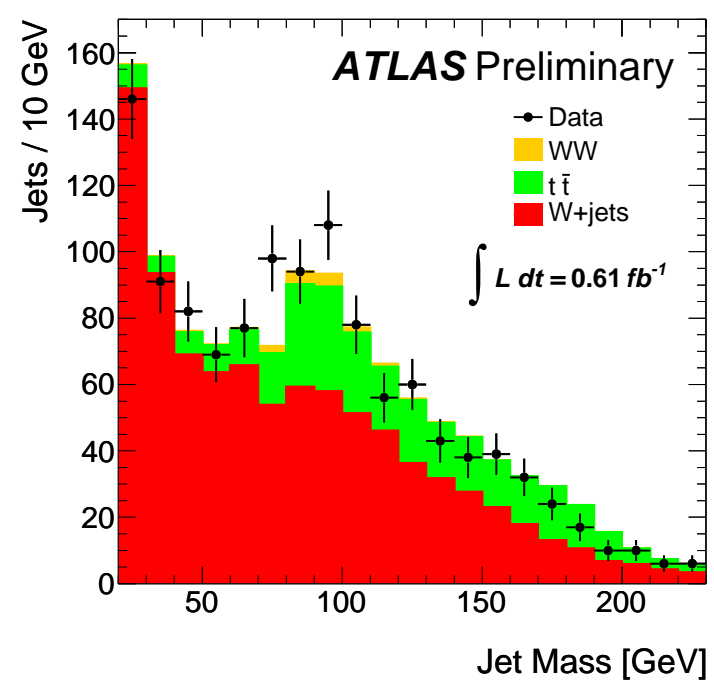

Figure 10: Jet mass in $W+$ jets for a jet with substructure compatible with $W Z(\rightarrow b b)$ or $W H(\rightarrow b b)$ events. Figure taken from ref. [100].

\section{Conclusions}

QCD is a dynamic field - there has been a spectacular progress in recent years. This includes amazing technical achievements (higher multiplicities and/or loops), clever merging procedures to catch the best features of different calculations, ingenuity in refining observables, sophisticated techniques for looking inside jets, and spectacular formal developments (IR/UV structures, $N=4$ or $N=8$ super Yang-Mills calculations, twistors developments, the connection between Wilson loops and amplitudes, symbols, ...), which I did not have time to mention.

Impressive results have already come out of the LHC, but this is certainly only the tip of the iceberg. We are well prepared to make the most out of the observations at the LHC. The challenge we however face is vast, so that it is more important than ever to choose the right observables and tools for a given physics analysis.

\section{Acknowledgments}

I am grateful to Ulrich Haisch, Tom Melia, and Gavin Salam for comments on this writeup and interesting discussions.

\section{References}

[1] T. Aaltonen et al. [CDF Collaboration], Phys. Rev. Lett. 106 (2011) 171801 [arXiv:1104.0699 [hep-ex]].

[2] http://www-cdf.fnal.gov/physics/ewk/2011/wjj/

[3] T. Plehn, M. Takeuchi, J. Phys. G G38 (2011) 095006 [arXiv:1104.4087 [hep-ph]].

[4] J. M. Campbell, A. Martin, C. Williams, Phys. Rev. D84 (2011) 036005 [arXiv:1105.4594 [hep-ph]]. 
[5] Z. Sullivan, A. Menon, [arXiv:1108.4676 [hep-ph]].

[6] V. M. Abazov et al. [ D0 Collaboration ], Phys. Rev. Lett. 107 (2011) 011804 [arXiv:1106.1921 [hep-ex]].

[7] T. Aaltonen et al. [ CDF Collaboration ], Phys. Rev. D83 (2011) 112003. [arXiv:1101.0034 [hep-ex]].

[8] V. M. Abazov et al. [ D0 Collaboration ], [arXiv:1107.4995 [hep-ex]].

[9] V. M. Abazov et al. [ D0 Collaboration ], Phys. Rev. D84 (2011) 052007. [arXiv:1106.6308 [hep-ex]].

[10] T. Aaltonen et al. [ CDF Collaboration ], Phys. Rev. Lett. 104 (2010) 131801. [arXiv:0909.1505 [hep-ex]].

[11] M. Moretti, F. Piccinini, R. Pittau, A. D. Polosa, JHEP 0307 (2003) 001 [hep-ph/0206293].

[12] M. Herquet, F. Maltoni, O. Mattelaer, T. Stelzer, JHEP 1106 (2011) 128 [arXiv:1106.0522 [hep-ph]].

[13] T. Gleisberg, S. .Hoeche, F. Krauss, M. Schonherr, S. Schumann, F. Siegert, J. Winter, JHEP 0902 (2009) 007 [arXiv:0811.4622 [hep-ph]].

[14] T. Sjostrand, S. Mrenna, P. Skands, Comput. Phys. Commun. 178 (2008) 852-867 [arXiv:0710.3820 [hep-ph]].

[15] S. Gieseke, D. Grellscheid, K. Hamilton, A. Papaefstathiou, S. Platzer, P. Richardson, C. A. Rohr, P. Ruzicka [arXiv:1102.1672 [hep-ph]].

[16] G. Aad et al. [ Atlas Collaboration ], [arXiv:1110.2299 [hep-ex]].

[17] V. Khachatryan et al. [ CMS Collaboration ], Phys. Lett. B699 (2011) 48-67. [arXiv:1102.0068 [hep-ex]].

[18] J. R. Andersen et al. [ SM and NLO Multileg Working Group Collaboration ], [arXiv:1003.1241 [hep-ph]].

[19] R. Britto, F. Cachazo, B. Feng, Nucl. Phys. B725 (2005) 275-305 [hep-th/0412103].

[20] G. Ossola, C. G. Papadopoulos, R. Pittau, Nucl. Phys. B763 (2007) 147-169 [hep-ph/0609007].

[21] W. T. Giele, Z. Kunszt, K. Melnikov, JHEP 0804 (2008) 049 [arXiv:0801.2237 [hep-ph]].

[22] R. K. Ellis, Z. Kunszt, K. Melnikov, G. Zanderighi, [arXiv:1105.4319 [hep-ph]].

[23] L. Dixon, these proceedings.

[24] R. K. Ellis, K. Melnikov, G. Zanderighi, Phys. Rev. D80 (2009) 094002 [arXiv:0906.1445 [hep-ph]].

[25] C. F. Berger, Z. Bern, L. J. Dixon, F. Febres Cordero, D. Forde, T. Gleisberg, H. Ita, D. A. Kosower et al., Phys. Rev. Lett. 102 (2009) 222001 [arXiv:0902.2760 [hep-ph]].

[26] Z. Bern, L. J. Dixon, F. Febres Cordero, D. Forde, T. Gleisberg, H. Ita, D. A. Kosower et al., Phys. Rev. D82 (2010) 074002 [arXiv:1004.1659 [hep-ph]].

[27] G. Bevilacqua, M. Czakon, C. G. Papadopoulos, R. Pittau, M. Worek, JHEP 0909 (2009) 109. [arXiv:0907.4723 [hep-ph]].

[28] M. Czakon, A. van Hameren, C. G. Papadopoulos, M. Worek, JHEP 1102 (2011) 083 [arXiv:1012.4230 [hep-ph]].

[29] T. Melia, K. Melnikov, R. Rontsch, G. Zanderighi, JHEP 1012 (2010) 053 [arXiv:1007.5313 [hep-ph]]. 
[30] T. Melia, K. Melnikov, R. Rontsch, G. Zanderighi, Phys. Rev. D83 (2011) 114043 [arXiv:1104.2327 [hep-ph]].

[31] G. Bevilacqua, M. Czakon, C. G. Papadopoulos, M. Worek, [arXiv:1108.2851 [hep-ph]].

[32] T. Binoth, N. Greiner, A. Guffanti, J. Reuter, J. -P. .Guillet, T. Reiter, Phys. Lett. B685 (2010) 293-296 [arXiv:0910.4379 [hep-ph]].

[33] A. Bredenstein, A. Denner, S. Dittmaier, S. Pozzorini, JHEP 1003 (2010) 021 [arXiv:1001.4006 [hep-ph]].

[34] A. Denner, S. Dittmaier, S. Kallweit, S. Pozzorini, Phys. Rev. Lett. 106 (2011) 052001 [arXiv:1012.3975 [hep-ph]].

[35] K. Arnold, J. Bellm, G. Bozzi, M. Brieg, F. Campanario, C. Englert, B. Feigl, J. Frank et al., [arXiv:1107.4038 [hep-ph]].

[36] C. F. Berger, Z. Bern, L. J. Dixon, F. Febres Cordero, D. Forde, T. Gleisberg, H. Ita, D. A. Kosower et al., Phys. Rev. Lett. 106 (2011) 092001 [arXiv:1009.2338 [hep-ph]].

[37] H. Ita, Z. Bern, L. J. Dixon, F. F. Cordero, D. A. Kosower, D. Maitre, [arXiv:1108.2229 [hep-ph]].

[38] V. Hirschi, R. Frederix, S. Frixione, M. V. Garzelli, F. Maltoni, R. Pittau, JHEP 1105 (2011) 044 [arXiv:1103.0621 [hep-ph]].

[39] G. Bevilacqua, M. Czakon, M. V. Garzelli, A. van Hameren, A. Kardos, C. G. Papadopoulos, R. Pittau, M. Worek, [arXiv:1110.1499 [hep-ph]].

[40] S. Frixione, F. Stoeckli, P. Torrielli, B. R. Webber, C. D. White, [arXiv:1010.0819 [hep-ph]].

[41] P. Nason, JHEP 0411 (2004) 040 [arXiv:hep-ph/0409146 [hep-ph]].

[42] S. Alioli, P. Nason, C. Oleari, E. Re, JHEP 1006 (2010) 043 [arXiv:1002.2581 [hep-ph]].

[43] T. Melia, P. Nason, R. Rontsch, G. Zanderighi, Eur. Phys. J. C71 (2011) 1670 [arXiv:1102.4846 [hep-ph]].

[44] R. Frederix, S. Frixione, V. Hirschi, F. Maltoni, R. Pittau, P. Torrielli, JHEP 1109 (2011) 061 [arXiv:1106.6019 [hep-ph]].

[45] R. Frederix, S. Frixione, V. Hirschi, F. Maltoni, R. Pittau, P. Torrielli, Phys. Lett. B701 (2011) 427-433 [arXiv:1104.5613 [hep-ph]].

[46] R. Frederix, S. Frixione, V. Hirschi, F. Maltoni, R. Pittau, P. Torrielli, [arXiv:1110.5502 [hep-ph]].

[47] K. Hamilton, P. Nason, JHEP 1006 (2010) 039 [arXiv:1004.1764 [hep-ph]].

[48] S. Alioli, K. Hamilton, E. Re, [arXiv:1108.0909 [hep-ph]].

[49] M. Rubin, G. P. Salam, S. Sapeta, JHEP 1009 (2010) 084 [arXiv:1006.2144 [hep-ph]].

[50] S. Catani, L. Cieri, G. Ferrera, D. de Florian, M. Grazzini, Phys. Rev. Lett. 103 (2009) 082001 [arXiv:0903.2120 [hep-ph]].

[51] R. Gavin, Y. Li, F. Petriello, S. Quackenbush, Comput. Phys. Commun. 182 (2011) 2388-2403 [arXiv:1011.3540 [hep-ph]].

[52] G. Bozzi, S. Catani, G. Ferrera, D. de Florian, M. Grazzini, Phys. Lett. B696 (2011) 207-213. [arXiv:1007.2351 [hep-ph]]. 
[53] Q. H. Cao, C. R. Chen, C. Schmidt and C. P. Yuan, arXiv:0909.2305 [hep-ph].

[54] S. Chatrchyan et al. [ CMS Collaboration ], [arXiv:1108.0566 [hep-ex]].

[55] G. Aad et al. [ATLAS Collaboration], arXiv:1110.5016 [hep-ex].

[56] J. Alcaraz, these proceedings.

[57] CMS collaboration, https://twiki.cern.ch/twiki/bin/view/CMSPublic/PhysicsResultsEWK.

[58] G. Aad et al. [ATLAS Collaboration], arXiv:1108.3699 [hep-ex].

[59] S. Chatrchyan et al. [CMS Collaboration], arXiv:1108.3773 [hep-ex].

[60] Y. Kiyo, J. H. Kuhn, S. Moch, M. Steinhauser, P. Uwer, Eur. Phys. J. C60 (2009) 375-386 [arXiv:0812.0919 [hep-ph]].

[61] V. Ahrens, A. Ferroglia, M. Neubert, B. D. Pecjak, L. L. Yang, [arXiv:1103.0550 [hep-ph]].

[62] M. Beneke, P. Falgari, S. Klein, C. Schwinn, [arXiv:1109.1536 [hep-ph]].

[63] N. Kidonakis, [arXiv:1109.3231 [hep-ph]].

[64] M. Czakon, Nucl. Phys. B 849 (2011) 250 [arXiv:1101.0642 [hep-ph]].

[65] M. Spira, A. Djouadi, D. Graudenz, P. M. Zerwas, Nucl. Phys. B453 (1995) 17-82. [arXiv:hep-ph/9504378 [hep-ph]].

[66] R. V. Harlander, W. B. Kilgore, Phys. Rev. Lett. 88 (2002) 201801. [hep-ph/0201206].

[67] C. Anastasiou, K. Melnikov, Nucl. Phys. B646 (2002) 220-256. [hep-ph/0207004].

[68] V. Ravindran, J. Smith, W. L. van Neerven, Nucl. Phys. B665 (2003) 325-366. [arXiv:hep-ph/0302135 [hep-ph]].

[69] S. Actis, G. Passarino, C. Sturm, S. Uccirati, Phys. Lett. B670 (2008) 12-17. [arXiv:0809.1301 [hep-ph]].

[70]

[70] C. Anastasiou, R. Boughezal, F. Petriello, JHEP 0904 (2009) 003. [arXiv:0811.3458 [hep-ph]].

[71] S. Catani, D. de Florian, M. Grazzini, P. Nason, JHEP 0307 (2003) 028. [hep-ph/0306211].

[72] S. Moch, A. Vogt, Phys. Lett. B631 (2005) 48-57. [hep-ph/0508265].

[73] E. Laenen, L. Magnea, Phys. Lett. B632 (2006) 270-276. [hep-ph/0508284].

[74] V. Ahrens, T. Becher, M. Neubert, L. L. Yang, Phys. Lett. B698 (2011) 271-274. [arXiv:1008.3162 [hep-ph]].

[75] C. Anastasiou, K. Melnikov, F. Petriello, Nucl. Phys. B724 (2005) 197-246. [hep-ph/0501130].

[76] M. Grazzini, JHEP 0802 (2008) 043. [arXiv:0801.3232 [hep-ph]].

[77] G. Ferrera, M. Grazzini, F. Tramontano, [arXiv:1107.1164 [hep-ph]].

[78] J. Baglio, A. Djouadi, JHEP 1010 (2010) 064. [arXiv:1003.4266 [hep-ph], arXiv:1009.1363 [hep-ph]].

[79] V. Ahrens, T. Becher, M. Neubert, L. L. Yang, Eur. Phys. J. C62 (2009) 333-353. [arXiv:0809.4283 [hep-ph]]. 
[80] S. Dittmaier et al. [ LHC Higgs Cross Section Working Group Collaboration ], [arXiv:1101.0593 [hep-ph]].

[81] ATLAS collaboration, ATLAS-CONF-2011-134.

[82] C. Anastasiou, G. Dissertori, M. Grazzini, F. Stockli and B. R. Webber, JHEP 0908 (2009) 099 [arXiv:0905.3529 [hep-ph]].

[83] C. Anastasiou, G. Dissertori, F. Stockli and B. R. Webber, JHEP 0803 (2008) 017 [arXiv:0801.2682 [hep-ph]].

[84] J. M. Campbell, R. K. Ellis and C. Williams, Phys. Rev. D 81 (2010) 074023 [arXiv:1001.4495 [hep-ph]].

[85] I. W. Stewart, F. J. Tackmann, [arXiv:1107.2117 [hep-ph]].

[86] G. Bozzi, S. Catani, D. de Florian and M. Grazzini, Nucl. Phys. B 737 (2006) 73 [arXiv:hep-ph/0508068].

[87] C. F. Berger, C. Marcantonini, I. W. Stewart, F. J. Tackmann and W. J. Waalewijn, JHEP 1104 (2011) 092 [arXiv:1012.4480 [hep-ph]].

[88] S. Alekhin, S. Alioli, R. D. Ball, V. Bertone, J. Blumlein, M. Botje, J. Butterworth, F. Cerutti et al., [arXiv:1101.0536 [hep-ph]].

[89] S. Bethke, A. H. Hoang, S. Kluth, J. Schieck, I. W. Stewart, S. Aoki, M. Beneke, J. Blumlein et al., [arXiv:1110.0016 [hep-ph]].

[90] S. Bethke, Eur. Phys. J. C64 (2009) 689-703. [arXiv:0908.1135 [hep-ph]].

[91] R. Abbate, M. Fickinger, A. H. Hoang, V. Mateu, I. W. Stewart, Phys. Rev. D83 (2011) 074021. [arXiv:1006.3080 [hep-ph]].

[92] A. Pich, Acta Phys. Polon. Supp. 3 (2010) 165 [arXiv:1001.0389 [hep-ph]].

[93] G. Abbiendi et al. [ OPAL Collaboration ], Eur. Phys. J. C71 (2011) 1733. [arXiv:1101.1470 [hep-ex]].

[94] R. D. Ball et al. [ The NNPDF Collaboration ], [arXiv:1107.2652 [hep-ph]].

[95] V. M. Abazov et al. [D0 Collaboration], Phys. Rev. D 80, 111107 (2009) [arXiv:0911.2710 [hep-ex]].

[96] G. Dissertori, A. Gehrmann-De Ridder, T. Gehrmann, E. W. N. Glover, G. Heinrich and H. Stenzel, Phys. Rev. Lett. 104 (2010) 072002 [arXiv:0910.4283 [hep-ph]].

[97] R. Frederix, S. Frixione, K. Melnikov, G. Zanderighi, JHEP 1011 (2010) 050 [arXiv:1008.5313 [hep-ph]].

[98] M. Cacciari, G. P. Salam, G. Soyez, JHEP 0804 (2008) 063 [arXiv:0802.1189 [hep-ph]].

[99] A. Abdesselam, et al., Eur. Phys. J. C71 (2011) 1661.

[100] Talk given by Ricardo Goncalo on behalf of the ATLAS collaboration at EPS 2011. 